\title{
Hinge Device
}

National Cancer Institute

\section{Source}

National Cancer Institute. Hinge Device. NCI Thesaurus. Code C49988.

A device designed to join two objects and allow them to swing relative to one another. 